\title{
Common basis for health risk assessment and safety decision-making concerning exposure to ionising radiation and other sources of harm
}

\author{
V.F. Demin \\ RRC “Kurchatov institute", Moscow, Russia
}

\begin{abstract}
To meet the growing practical need in risk analysis in Russia health risk assessment tools and regulations have been developed in the frame of few federal research programs. One of the objectives and directions of this development is to produce common, unified basis of health risk analysis for different sources of risk. A specific risk index $\Re$ has been proposed for safety decision-making (establishing safety standards and other levels of protective actions, comparison of various sources of risk, etc.). The index $\Re$ is defined as the partial mathematical expectation of lost years of healthy life (LLE) due to exposure during a year to a risk source considered. The more concrete determinations of this index for different risk sources derived from the common definition of $\mathfrak{R}$ are given. Generic safety standards (GSS) for the public and occupational workers have been suggested in terms of this index. Secondary specific safety standards have been derived from GSS for ionising radiation and a number of other risk sources including environmental chemical pollutants. Other general and derived levels for decision-making have also been proposed including the "de-minimus" level. Some examples of risk comparison are demonstrated in the frame of different comparison tasks.
\end{abstract}

\section{INTRODUCTION}

In Russia needs in practical use of health risk (HR) analysis began to rise since the end of 80s. They came from decision making on the rehabilitation, radiation and social protection of population on the territories suffered from the Chernobyl and other radiation accidents, nuclear weapon tests, etc. Demands of the current ecological regulation concerning the development of the hazardous industries, especially the fuel-power complex, appeal also to risk analysis. In 1997 Public Health Ministry of Russia issued the special decree on development and use of HR analysis in decision-making on environment and public health protection.

To meet these needs the development of HR analysis means and regulation began in the frame of few research state programs. RRC "Kurchatov Institute" have been involved in these R \& D. The directions of this activity are: development of HR assessment tool (methodology, computer modules and data bases), regulatory aspects of use of risk analysis in decision-making, case studies, etc. Both radiation and non-radiation $\mathrm{HR}$, including risk from chemical contamination of the environment, have been considered. The tool includes methodology and computer module of HR assessment. The module was called BARD: bank of data for assessment of risk. The tool has been continuously developed using new achievements and responding to practical demands [1- 4]. One of the objectives and directions of this development is to produce common, unified basis of HR analysis for different sources of risk. Application areas of the tool are:

- $\quad$ assessment of the radiological and non-radiological consequences of radiation accidents and nuclear weapons tests; 
- assessment of the radiation risk from any source of radiation exposure (natural, medical and technogenic);

- $\quad$ HR assessment and comparison for different energy systems;

- $\quad$ analysis of the public health in terms of risk and health-demographic indices;

- assessment of HR from other technogenic sources (if input data are available, e.g. risk from chemical air pollution).

The paper is based on some results of R \& D mentioned above. Partly these R \& D have been carried out in the frame of the project \# 2558 of the International Science-Technology Center (Moscow, Russia).

\section{COMMON METHODICAL BASIS}

In the past main attention was paid to radiation risk. The hazard estimation and establishment of ecological and safety standards for chemical contaminants of the environment were based on the toxicology and threshold exposure - response function (ERF) approach. In 90s it was recognised that for some chemical substances non-threshold ERF and risk assessment should be added into the health hazard study and safety decision-making. It concerns chemical carcinogens and some non-carcinogenic chemical substances, e.g. the environment contaminants from the fossil fuel power plants: particulate matters (PM), $\mathrm{SO}_{2}, \mathrm{NO}_{\mathrm{x}}$ and their secondary pollutants [5].

A common basis for risk assessment and decision making on population health protection should be developed and used. This common basis should include:

1. general method of HR assessment,

2. ERF (for a risk source considered) produced in the form of age-cause-specific mortality or morbidity rate,

3. country or regional health-demographic data (HDD).

Only all these three parts can allow to calculate any necessary set of specific or general HR indices in all possible dependencies and then in averaged forms if it is needed.

Point 1 is well known in the methodology of risk assessment. HDD can be easily received from a state statistic department or from WHO database. All difficult problems are connected with point 2 .

Health effects of ionising radiation and many chemical contaminants are non-specific. ERF can be obtained only by carrying out epidemiological studies. As a rule, such studies for low exposures are very complicated, long-term and expensive. In many cases they have a limited statistical power.

An important factor in HR quantification is how to transfer site-specific risks across populations. In other words, how risk coefficients estimated for one population apply to another population with different HDD [6].

For ionising radiation and some limited number of chemical pollutants ERFs were obtained on the basis of results of epidemiological studies and were presented in the form mentioned above (point 2). Radiation risk models like BEIR V, VI [7,8], UNSCEAR-94, 2000 [6,9] are of such form. The risk models for chemical pollutants $\mathrm{PM}, \mathrm{SO}_{2}, \mathrm{NO}_{\mathrm{x}}$ with their secondary pollutants have the same form $[5,11,12]$.

The most part of these ERFs is of multiplicative risk mode dependence: excess incidence rate of health effects is proportional to baseline rates ("spontaneous" rates). In this case the transfer across populations is made not of ERF total, but only relative risk coefficients. If such dependence is not only a methodical approach and it reflects the medical-biological nature of formation of the health effect, it can be a good argument for the validity of the transfer.

If risk assessment is made for some specific population and the results are presented in an averaged form it is not generally valid to transfer these results to another population.

\section{SPECIAL RISK INDEX AND DECISION MAKING LEVELS}

Up to now there is no common approach in establishment of health protective levels for different sources of risk. 
To protect population or personnel from dangerous industry activities the risk limits are usually established in the terms of individual mortality rate $r$. It is obvious that such limits can't be properly used for the risk sources with delayed health effects (ionising radiation, impact of some chemicals especially with carcinogenic or hereditary effects, etc.).

In radiation protection the generalized risk index $R_{E}$ is constructed and used to produce such quantity as the effective dose for establishing safety standards [10]. It is not so simple to use this index for comparison of HR for ionising radiation and other risk sources and to compare radiation and non-radiation safety standards. Now the use of HR assessment in establishing safety standards for some chemical contaminants is in the progress [12]. What risk index could be used in this case?

Cases of death from different sources of risk can differ considerably by the damage expressed in the loss of life expectancy (LLE). For chemical and radiation carcinogenesis there is a relatively large latent time interval $T_{l}$ (between an exposure and appearance of a cancer): for solid cancers the minimal value of $T_{l}$ is equal to 5-10 years, averaged one $-40-50$ years. Due to this the averaged LLE $\left(L_{a v}^{\text {l.c. }}\right)$ for one lethal radiogenic cancer is considerably less than averaged LLE $\left(L_{a v .}^{i . d .}\right)$ due to immediate death from an accident or adverse effect. Analogous situation is for the chemical carcinogenesis.

In the accordance with the ICRP estimation [10] the global values of these quantities are equal to

$$
\begin{aligned}
& L_{a v .}^{\text {l.c. }}=15 \text { years }, \\
& L_{\text {av. }}^{\text {i.d. }}=35 \text { years } .
\end{aligned}
$$

Values of LLE (specific or averaged) from sources of risk can be estimated for population of any country or region using a computer code like BARD. The risk coefficient of the effective dose $g_{E}[10]$ (the globally averaged specific LLE for ionising radiation), which included all health effects (cancer mortality and morbidity, hereditary effects), is equal to

$$
g_{E}=\left\{\begin{array}{l}
1 \text { man } \cdot y r / m a n \cdot S v \text { for public, } \\
0.8 \text { man } \cdot y r / m a n \cdot S v \text { for professional exposure. }
\end{array}\right.
$$

After analyzing the aspects of establishment of safety standards, decision making levels, the risk indices and ways of their integration for different risk sources, especially ICRP recommendation [10], the proposal was made concerning the special risk index $\mathscr{R}$ for the risk standardization and comparison [2,3]. $\mathscr{R}$ is quantitatively defined as LLE caused by the annual exposure to the risk source considered:

$$
\mathfrak{R}=\text { annual exposure } * \text { damage (LLE) from the exposure unit. }
$$

For different risk sources the exposure can be measured with different quantities. For immediate death due to an accident or an adverse effect the exposure is expressed in the term of the probability of death. In this concrete case

$$
\Re=r \cdot g_{r} .
$$

Here $r$ is the individual death rate (in other words, annual probability of death), $g_{r}$ is LLE due to a death in the year considered.

For ionising radiation dose $D$ (absorbed, equivalent or effective - in the dependence of the type of radiation and an application area) is used as a measure of exposure. For this case $\mathfrak{R}$ is expressed as

$$
\Re=d_{\text {i.r. }} \cdot g_{\text {i.r. }} \text {, }
$$

where $d_{i . r .}$ is the ionizing radiation dose rate, $g_{i . r .}$ is LLE from the dose unit.

As a rule, ERFs for chemical contaminants are referred to exposure $\varepsilon_{c h}$ - the time integral of a concentration of a contaminant in the air or water. The dimension of $\varepsilon_{c h}$ is $\left[\mathrm{yr} * \mu \mathrm{g} / \mathrm{m}^{3}\right]$. The expression (4) for $\mathfrak{R}$ should be rewritten in the following concrete form: 


$$
\Re=\boldsymbol{e}_{c h} \cdot g_{c h}
$$

$e_{c h}$ is the exposure rate (the annual exposure); its dimension is $\left[\left(\mathrm{yr} * \mu \mathrm{g} / \mathrm{m}^{3}\right) / \mathrm{yr}\right] ; g_{c h}$ is LLE from the exposure unit.

The meaning of $\mathscr{R}$ can be seen from Eqs. (4) - (7). It is relative LLE: LLE in years referred to 1 year under the exposure to a risk source. The dimension of this value is [yr(LLE)/yr]. In the statistical sense $\mathscr{R}$ is conditionally the share of the year, which is lost due to the exposure to a risk source during this year. In this sense $\mathscr{R}$ can be called as the relative damage. Really lifetime years are lost after the exposure. Taking into account this comment $\mathfrak{R}$ can be in some conditional sense considered as a dimensionless quantity.

The definitions (4) - (7) can be also generalised for morbidity.

In establishing the health protection standards and levels it is reasonable to use the averaged values in Eqs (5) - (7) as it was done, e.g., in the radiation protection for radiation risk coefficients [10]. In average through age $g_{r}=L_{a v .}^{i . d .}=35$ years, see Eq. (2). In Eq. (6) the effective dose and its coefficients can be used:

$$
\Re=d_{E} \cdot g_{E},
$$

where $d_{E}$ is effective dose rate (annual effective dose), $g_{E}$ is well-known LLE risk coefficient for the ionising radiation, see Eq. (3).

From the literature one can find some important examples for the chemical airborne pollutants:

$$
g_{c h}=\left\{\begin{array}{l}
0.0006 \mathrm{yr} / \mathrm{yr} \cdot \mu \mathrm{g} / \mathrm{m}^{3} \text { for } \mathrm{PM}_{2.5} \text { and EU population [11], } \\
0.0008 \mathrm{yr} / \mathrm{yr} \cdot \mu \mathrm{g} / \mathrm{m}^{3} \text { for } \mathrm{PM}_{2.5} \text { and Russia population [11], } \\
0.002 \mathrm{yr} / \mathrm{yr} \cdot \mu \mathrm{g} / \mathrm{m}^{3} \text { for As and global population [12]. }
\end{array}\right.
$$

Cancer is considered as the health effect from air pollutant As. For $\mathrm{PM}_{2.5}$ health effects are diseases of respiratory and circulatory systems and possible death from them.

Using risk index $\mathscr{R}$ the universal safety standards (risk limits $\mathfrak{R}_{n}$ ) can be established. To have them being formally equivalent to radiation safety basic standards [10]

$$
d_{E, n}=\left\{\begin{array}{l}
1 \mathrm{mSv} / \mathrm{yr} \text { for public, } \\
20 \mathrm{mSv} / \mathrm{yr} \text { for personnel. }
\end{array}\right.
$$

one should adopt the following value (see Eqs (8), (3) and (10)):

$$
\mathfrak{R}_{n}=\left\{\begin{array}{l}
0.001 \text { for public, } \\
0.016 \text { for personnel. }
\end{array}\right.
$$

It is well known that some precaution was laid in ICRP effective dose limits (10). In calculation of risk index $R_{E}$ and its components the correction factor (DDREF) for the dependence of risk upon the dose and dose rate of the low-LET radiation was taken to be equal to 2 from uncertainty interval $2-10$ [10]. Due to this adoption some overestimation of the radiation risk was made in calculation of $R_{E}$ (in DDREF/2 times). It means that the dose limits (10) are really more rigid than the risk limits (11).

Taking into account this fact and necessity to deal with all regulated risk sources the following universal risk limits are proposed for consideration by the specialists in health risk analysis and members of regulatory bodies:

$$
\mathfrak{R}_{n}=\left\{\begin{array}{c}
0.0007 \text { for public, } \\
0.01 \text { for personnel. }
\end{array}\right.
$$


Basing on these general risk limits (safety standards) derivative limits for different risk sources can be obtained. For risk sources with non-delayed health effects (accident and adverse effects) the derivative risk limits in the terms of death risk rate $r$ are equal $\left(r_{n}=\Re_{n} / g_{r}, g_{r}=L_{a v}^{i . d}\right.$. , see Eqs (12), (2), (5))

$$
r_{n}= \begin{cases}2.0 \cdot 10^{-5} / \mathrm{yr} & \text { for public, } \\ 2.9 \cdot 10^{-4} / \mathrm{yr} & \text { for personnel. }\end{cases}
$$

For the chemical air pollutants considered above the derivative health protection standards in the term of the annually averaged air concentration $c_{n}$ should be $\left(c_{n}=\Re_{n} / g_{c h}\right.$, see Eqs (9) and (12)):

$$
c_{n}=\left\{\begin{array}{l}
1.2 \mu \mathrm{g} / \mathrm{m}^{3} \text { for } \mathrm{PM}_{2.5} \text { and EU population, } \\
0.9 \mu \mathrm{g} / \mathrm{m}^{3} \text { for } \mathrm{PM}_{2.5} \text { and Russia population, } \\
0.4 \mu \mathrm{g} / \mathrm{m}^{3} \text { for As and global population. }
\end{array}\right.
$$

It is also simple to calculate the derivative chemical safety standards for personnel in the terms of maximum permissible concentration $c_{n}$ from the definitions (9) and (12).

"De minimus" level $\mathfrak{R}_{\text {d.m. }}$ is proposed to be established as $\mathfrak{R}_{\text {d.m. }}=10^{-5}$. Accordingly the secondary "de minimus" levels should be $r_{\text {d.m.. }}=3 * 10^{-7} /$ year (risk sources with non-delayed health effects) and $20 \mu \mathrm{Sv} /$ year (ionizing radiation).

One should note the effect of competition of different risks. A man dies only once. This manifests itself in the fact that the total lifetime risk is equal to unity. A change in one of the death causes automatically leads to a change (renormalization) in the lifetime risk indices of other sources in action, even though they are statistically independent. These risk indices, as many others are not additive. Annual risks considered mutually compete with themselves, decreasing each other. However for impacts on the level of health protection standards the effect of the risks competition can be ignored. Moreover this ignorance gives an element of precaution (a reserve of reliability) in the establishing the safety standards, although it is rather insignificant.

The quantity, which possesses the additive property, is the age-cause-specific mortality.

\section{RISKS COMPARISON}

There are several different tasks for risks comparison, which can be met in papers published. The following one's have been met more often:

1. Comparison of the individual risk rate from different risk sources, e.g., risk from transports, nature disasters, risk from hazardous industrial plants, etc.

2. Comparison of safety standards for risk sources, approved on the basis of different approaches, e.g., radiation and chemical protection standards.

3. Comparison of risk sources by the total risk (damage), e.g., different power production systems.

4. Comparison of population health risks from different diseases or any other sources of risk for different regions of Russia, different countries, etc.

Not always the proper risk indices have been chosen for such comparisons. E.g., individual death rate (annual probability of death) often used is not valid for radiation exposure: annual exposure in a current year can cause a radiological cancer few decades later, but annual probability of death in a current year can be caused by an exposure during previous years. As it was explained above a number of death can be non-proper damage index for comparison of different sources. In such comparison LLE is more suitable. In ExternE project comparisons namely LLE is usually used [5]. 
Here an example of the individual risk comparison (task 1) is given. It is known that in Russia the probability of death caused by lightning blows and motor-transport accidents is in average equal to $10^{-7} / \mathrm{yr}$ and $2 \cdot 10^{-4} / \mathrm{yr}$, correspondingly. Annual effective dose outside the protection zone of a nuclear power plant (NPP) is not higher than $0.01 \mathrm{mSv} / \mathrm{yr}$. Then the individual risk from these sources of risk in the terms of quantity $\Re$, proposed above is equal to

$$
\mathfrak{R}= \begin{cases}4 \cdot 10^{-6} & - \text { lightning blows; } \\ 7 \cdot 10^{-3} & \text { - motor-transport accidents; } \\ \leq 10^{-5} & \text { - living near the NPP protection zone } .\end{cases}
$$

Let one consider the comparison of health risk, e.g., from spontaneous fatal cancers for France and Russia (task 4). As it follows from data available values of $\mu(a)$ - age-cause-specific cancer mortality rate - are rather close to each other in France and Russia: the differences are not higher than $20-30 \%$. What HR index can be properly used for the comparison?

Let it be the lifetime risk $R$. The calculation (with using BARD) gives the following results: the value of $R$ for Russia in $1.5-2$ times less (in dependence upon the cancer type), than for France. The same results would be obtained for radiological cancers and with other averaged or lifetime risk indices. The reason of such results is the following: the life expectancy in Russia is considerably less then in France (for male the difference is more than 10 years). In other words this fact can be explained by the risks competition. If some risk factors begin to increase (in Russia they are the circulatory system diseases and mortality from accidents and adverse effects), then value of $R$ for the risk source considered will decrease under the condition that its $\mu(a)$ is not changed.

If the comparison is made in the terms of the standardized annual mortality $m_{s}$ (averaged through the standard age distribution), then the rather close results are obtained for France and Russia: for male of France and Russia $m_{s} \approx 300$ (per $10^{5}$ people), for female $m_{s} \approx 150$ (Russia) and 170 (France). In the calculation with BARD the European age distribution standard, adopted by WHO, is used.

It should be clear that the last comparison is more valid than the previous one's. Quantity $m_{s}$ is one of the principal indices of the health - demographic analysis. But this quantity is not also a universal index for comparisons. It has its own limitations. Its value depends upon the choice of the standard.

The shortcoming of the HR indices connected with the effect of the risks competition can have an influence upon the possibility to find the optimal protection against the complex of risk factors [2].

Several other demonstrative examples of the risks comparison were presented in [3].

\section{CONCLUSION}

The special risk index $\Re$ is proposed for safety decision-making. General safety standards for public and occupational workers are suggested in terms of this index. Secondary safety standards have been derived for ionising radiation and a number of other risk sources including environmental chemical pollution. Using this and some other risk indices in different tasks of risks comparison is analysed.

The averaged values of risk indices are usually taken in establishing decision-making levels. In particular, the coefficients of the effective dose, which is used in the definition of the effective dose itself and the radiation safety standards, are based on the globally averaged lifetime risk indices. These averaged values are also used in the secondary safety standards proposed in the paper.

However, in the consideration of issues of risks comparisons, it was noted that the averaged and lifetime risk indices are not always properly reflecting the real impact of the risk sources due to the effects of risks competition. It makes critically analyze the use of averaged and lifetime risk indices in the different safety decision levels adopted.

The proposal on the special standard population has been elaborated to overcome this problem. Some ideas along this line can be found in [2]. 


\section{References}

[1] V.F. Demin, P. Hedemann-Jensen, in Proceedings of 10-th annual SRA-E meeting, Stockholm, 1997 (Stockholm School of Economics, 1997), p. 834.

[2] V.F. Demin, Atomnaya Energiya, 86, 46 (1999).

[3] V.F. Demin, V.Ya. Golikov, E.V. Ivanov, et al., Atomnaya Energiya, 90, 385 (2001).

[4] A.P. Vasiliev, V.F. Demin, in Proceedings of the International Conference "PSAM 5 - Probabilistic Safety Assessment and Management”, Osaka, Japan, 2000, edited by S. Kondo, K. Funita (Universal Academy Press, Tokyo, 2000), p. 281.

[5] Externalities of Fuel Cycles: "ExternE Project". V. 8, European Commission, DG XII, 1998.

[6] Sources and effects of ionising radiation, UNSCEAR 2000 report to the General Assembly, UN, New York, 2000.

[7] Health effects of exposure to low levels of ionising radiation (BEIR V), National Academy Press, Washington, D.C., 1990.

[8] Health effects of Exposure to Radon (BEIR VI), National Academy Press, Washington, D.C., 1999.

[9] Sources and effects of ionising radiation, UNSCEAR 1994 report to the General Assembly, UN, New York, 1994.

[10] ICRP, Recommendations of the Commission - 1990, ICRP Publication 60, Ann. ICRP, 21 (1991).

[11] L. Leksell, A. Rabl, Risk Analysis, 21, 35 (2001).

[12] S.M. Novikov, G.I. Rumyantsev, Z.I. Zoldakova, et al., Hygiene \& Sanitation, \# 1, 12 (1998). 